\title{
Observation of ion beam induced magnetic patterning using off-specular polarized neutron reflectometry
}

\author{
N. D. Telling a) \\ Joule Physics Laboratory, Institute for Materials Research, University of Salford, \\ Salford M5 4WT, United Kingdom \\ S. Langridge and R. M. Dalgliesh \\ Rutherford Appleton Laboratory, Chilton, Didcot, Oxfordshire OX11 OXQ, United Kingdom \\ P. J. Grundy and V. M. Vishnyakov \\ Joule Physics Laboratory, Institute for Materials Research, University of Salford, \\ Salford M5 4WT, United Kingdom
}

(Presented on 13 November 2002)

\begin{abstract}
The long-range magnetic structure in Co/Pt multilayers magnetically patterned by ion irradiation is observed by off-specular polarized neutron reflectivity. While both specular and off-specular measurements indicate the formation of an artificial domain structure when the sample is in its remanent state, resonant peaks seen in the diffuse scatter reveal long-range magnetic ordering with periodicity in agreement with the design value. These peaks are completely suppressed when the sample is saturated in plane, confirming their origin in the magnetic patterning of the multilayer.

(c) 2003 American Institute of Physics. [DOI: 10.1063/1.1544496]
\end{abstract}

\section{INTRODUCTION}

The drive to obtain higher density magnetic recording media has generated growing interest in patterned magnetic films as a possible solution to the thermally excited, spontaneous reversal of small magnetic grains (the so-called superparamagnetic limit). However, practical difficulties arising from the rough surface created by physical patterning have lead recently to the study of possible mechanisms for magnetically patterning films. One such approach is the use of ion beams to alter the microstructure of a previously prepared film.

Ion irradiation of $\mathrm{Co} / \mathrm{Pt}$ multilayer and sandwich structures has been shown previously to rotate the easy magnetization direction from out of plane to in plane. ${ }^{1-7}$ It has been found that this reorientation of the anisotropy is correlated with structural reordering of the buried multilayer interfaces ${ }^{2,4}$ and is accompanied by an increase in grain size. ${ }^{1,4}$ The relaxation of strain-induced perpendicular anisotropy is therefore likely to play a role in this reorientation transition. By irradiating through a suitable stencil, this effect can be utilized to create localized in-plane magnetization regions in an otherwise perpendicularly magnetized film. ${ }^{3-7}$ Ion irradiation through a periodic mask (i.e., a grating or mesh structure) will thus define a periodic artificial domain structure in the film.

Off-specular polarized neutron reflectivity (PNR) is an ideal probe of long-range lateral magnetic periodicity. Recently this technique has been used to study artificially prepared arrays of mesoscopic ferromagnetic disks. ${ }^{8}$ In this case there exists a structural as well as magnetic component to the periodicity. However, the technique also has a huge potential for probing the long-range order in structures where only the

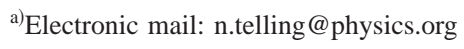

magnetic component changes, such as magnetically patterned films. As an experiment of this type, we discuss here measurements of the off-specular PNR scattering from a magnetically patterned $\mathrm{Co} / \mathrm{Pt}$ multilayer.

\section{EXPERIMENT}

$\mathrm{Co} / \mathrm{Pt}$ multilayers were prepared by magnetron sputtering using 3 mTorr partial pressure of $\mathrm{Kr}$ in a high-vacuum system with base pressure of $<\sim 10^{-7}$ Torr. The nominal structure of these films was $\operatorname{Si}(100) / \operatorname{Pt}(250 \AA) /$ $[\mathrm{Co}(4 \AA) / \mathrm{Pt}(13 \AA)]_{10}$. X-ray reflectivity measurements were used to confirm the layering sequence and gave the root mean square (rms) interface width between the Co and $\mathrm{Pt}$ layers as $\sim 4 \AA$. Ion-beam irradiation using a dose of $10^{15}$ ions $/ \mathrm{cm}^{2}$ Ar ions at $80 \mathrm{keV}$ was then performed simultaneously for larger areas of film and through a grid stencil with a period of $13 \mu \mathrm{m}$. In-plane and out-of-plane magnetic hysteresis loops were obtained from as-deposited and irradiated sections using alternating gradient field magnetometry (AGFM) and the magneto-optical Kerr effect (MOKE).

PNR experiments were performed on the CRISP reflectometer at the ISIS spallation neutron source, Rutherford Appleton Laboratory. Measurements were made at room temperature in a magnetic field applied parallel to the film plane and perpendicular to the neutron scattering plane. The sample was positioned such that design periodicity of $13 \mu \mathrm{m}$ occurred along an axis parallel to the neutron scattering plane. Off-specular scattering was measured using a position-sensitive detector.

\section{RESULTS AND DISCUSSION}

The as-deposited multilayers showed perpendicular anisotropy with coercivity of $\sim 1 \mathrm{kOe}$, as shown by the perpendicular loop in Fig. 1. After ion irradiation the magnetization 


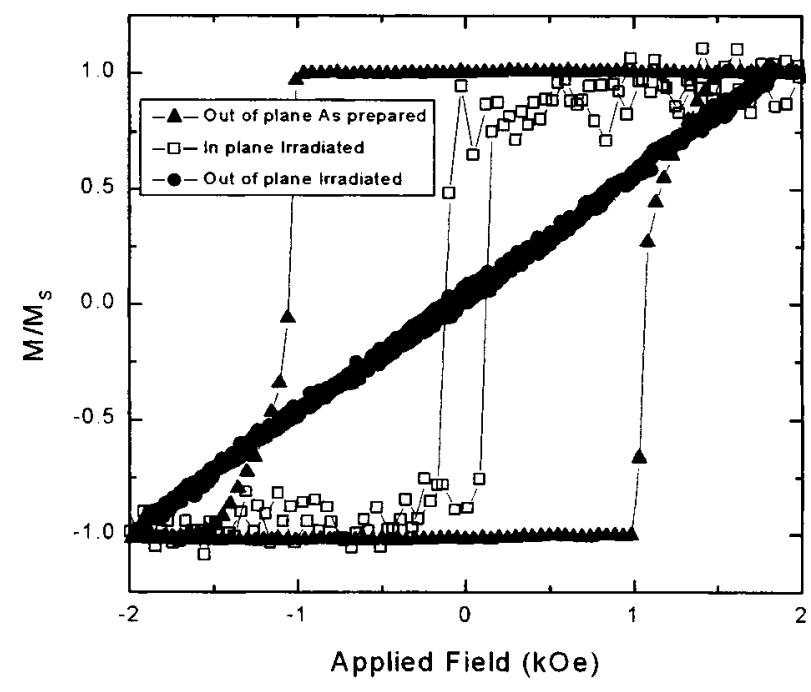

FIG. 1. Observed $M-H$ loops for the as-deposited multilayer (triangles, AGFM measurement) and ion beam irradiated multilayer (MOKE measurement).

was found to lie in the film plane and the coercivity was $\sim 200$ Oe (Fig. 1). Thus in the remanent state, after in-plane saturation, the irradiated regions in the patterned film will remain saturated along the axis of the field applied (i.e., in a single domain state) while the as-deposited regions will contain perpendicular domains. This multidomain nature of the as-deposited regions was confirmed using magnetic force microscopy (MFM). A schematic diagram showing the periodic artificial domain structure in the film and the neutron scattering arrangement is shown in Fig. 2. The interaction cross section between neutron moments and perpendicular film moments is zero. Thus, in the remanent state, the neutron scattering length will vary in a periodic fashion along the lateral direction. By measuring the off-specular neutron scattering, the in-plane component of the scattering vector, $q$, is used to probe this artificial periodicity.

The specular PNR curves obtained from the patterned film in its remanent state $(H \sim 40 \mathrm{Oe})$ and at saturation $(H$ $\sim 3 \mathrm{kOe}$ ) are shown in Figs. 3(a) and 3(b), respectively. The contribution to the measured moment comes only from the in-plane moments and is correlated with the degree of spin splitting of the reflectivity. The increase in the separation of spin states at saturation indicates the presence of both inplane and out-of-plane moments in the remanent state, consistent with the artificial domain structure illustrated in Fig. 2. The fits to the data shown in Fig. 2 indicate that the effec-

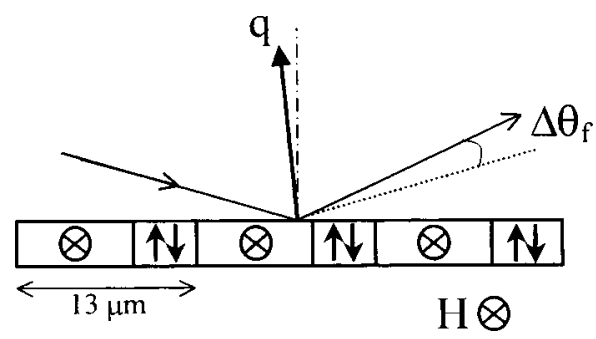

FIG. 2. Schematic of the ion-beam synthesized artificial magnetic domain structure in the film, showing also the neutron scattering geometry.

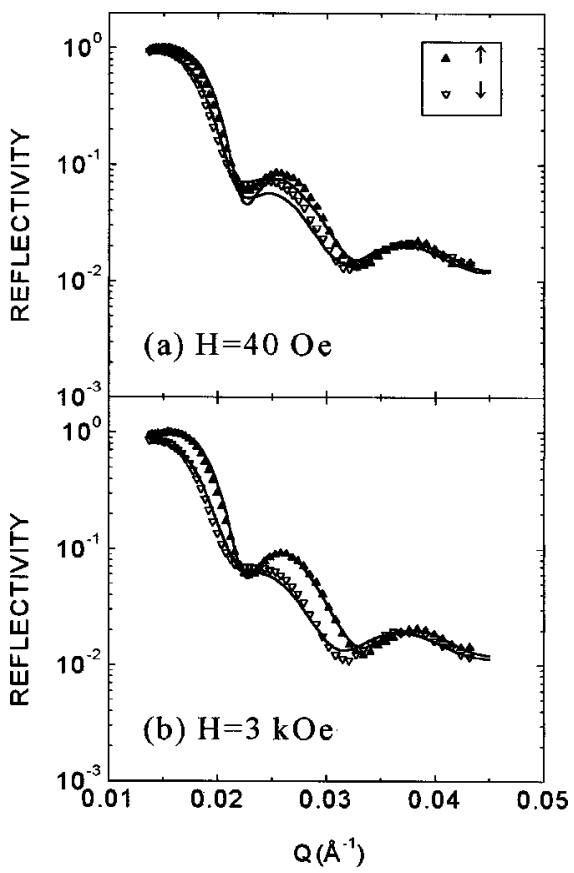

FIG. 3. Specular polarized neutron reflectivity measured at (a) remanence $(H \sim 40 \mathrm{Oe})$ and (b) saturation in plane $(H \sim 3 \mathrm{kOe})$.

tive moment increases from 0.6 to $1.9 \pm 0.15 \mu_{B}$ when the out-of-plane moments are forced into the plane at saturation.

Off-specular reflectivity was measured as a function of $q_{z}$ and the off-specular scattering angle, $\Delta \theta_{f}$, using the position-sensitive detector. The diffuse intensity measured at remanence $(H \sim 40 \mathrm{Oe})$ for spin-down reflectivity, integrated over $q_{z}$, is shown in Fig. 4. Clear resonant diffuse peaks can be seen on either side of the specular peak $\left(\Delta \theta_{f}=0\right)$. These peaks must result from very well defined periodicity which is indicative of the artificial period induced by the ion irradiation. Similar but weaker peaks were observed in the spin-up reflectivity (not shown), revealing the contrast in spindependent neutron scattering between the in-plane magnetic (irradiated) regions and out-of-plane (as-deposited) regions.

Interfacial roughness in multilayers results in a diffuse background contribution to the reflectivity that contains resonant features if any replication of interface roughness occurs. ${ }^{9}$ The interface modification caused by ion irradiation

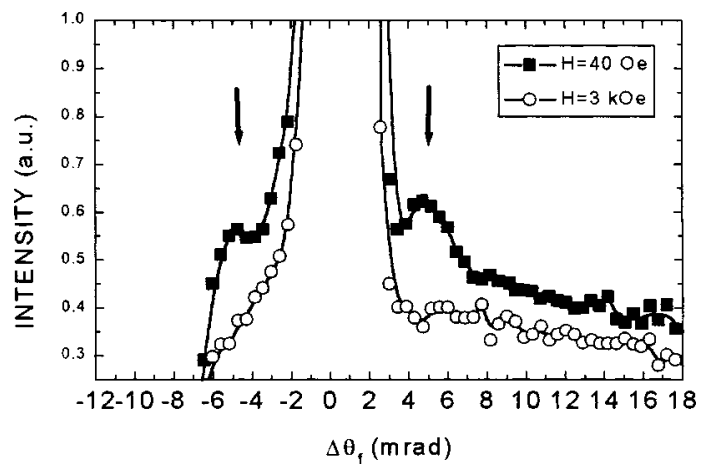

FIG. 4. Diffuse reflectivity, integrated over $q_{z}$, measured at remanence (40 $\mathrm{Oe})$ and at saturation $(3 \mathrm{kOe})$. 
through the stencil will lead to lateral structural periodicity that modulates the structural diffuse scattering, and could cause peaks such as those observed in Fig. 4. However, as a consequence of the smooth uncorrelated interfaces in this sample, a negligible diffuse background from the structural scattering was observed, and the peaks seen in Fig. 4 must therefore be due to magnetic diffraction.

The position of the resonant peaks corresponds well with that expected from the design period of $13 \mu \mathrm{m}$ (indicated by the arrows in Fig. 4). Observation of these peaks reveals the long-range magnetic order that has been induced in the film. From the schematic shown in Fig. 2 it can be seen that when the film is saturated in plane, thus forcing the out-of-plane moments in plane, the artificial magnetic domain structure will be erased and the periodicity lost. This is seen by the complete suppression of the resonant diffuse peaks in the diffuse curve measured at $3 \mathrm{kOe}$, shown in Fig. 4. Significantly, this confirms the magnetic rather than physical nature of the patterning induced by ion irradiation. This is consistent with our previous study ${ }^{4}$ which found that appreciable surface roughening/etching did not occur for the dose used here $\left(10^{15}\right.$ ions $\left./ \mathrm{cm}^{2}\right)$.

\section{CONCLUSION}

Off-specular polarized neutron reflectivity was used to probe a $\mathrm{Co} / \mathrm{Pt}$ multilayer that was magnetically patterned using ion irradiation through a stencil. Both specular and off- specular measurements indicate the formation of an artificial magnetic domain structure when the sample is in its remanent state. Resonant peaks in the off-specular curves convey long-range magnetic ordering with periodicity that is in agreement with the design value. The complete suppression of the peaks when the sample is saturated in plane confirm that purely magnetic rather than physical patterning of the film has occurred.

\section{ACKNOWLEDGMENT}

The authors would like to thank Dr. M. J. Bonder for preparing the $\mathrm{Co} / \mathrm{Pt}$ multilayer samples.

${ }^{1}$ R. Hyndman, P. Warin, J. Gierak, J. Ferré, J. N. Chapman, J. P. Jamet, V. Mathet, and C. Chappert, J. Appl. Phys. 90, 3843 (2001).

${ }^{2}$ D. Weller et al., J. Appl. Phys. 87, 5768 (2000).

${ }^{3}$ C. Chappert et al., Science 280, 1919 (1998).

${ }^{4}$ M. J. Bonder, N. D. Telling, P. J. Grundy, C. A. Faunce, and V. M. Vishnyakov Proceedings of the 47th Magnetism and Magnetic Materials Conference, 2002.

${ }^{5}$ B. D. Terris, L. Folks, D. Weller, J. E. E. Baglin, A. J. Kellock, H. Rothuizen, and P. Vettiger, Appl. Phys. Lett. 75, 403 (1999).

${ }^{6}$ B. D. Terris, D. Weller, L. Folks, J. E. E. Baglin, A. J. Kellock, H. Rothuizen, and P. Vettiger, J. Appl. Phys. 87, 7004 (2000).

${ }^{7}$ P. Warin, R. Hyndman, J. Glerak, J. N. Chapman, J. Ferré, J. P. Jamet, V. Mathet, and C. Chappert, J. Appl. Phys. 90, 3850 (2001).

${ }^{8}$ K. Temst, M. J. Van Bael, and H. Fritzsche, Appl. Phys. Lett. 79, 991 (2001)

${ }^{9}$ S. Langridge, J. Schmalian, C. H. Marrows, D. T. Dekadjevi, and B. J. Hickey, Phys. Rev. Lett. 85, 4964 (2000). 